\title{
Colecistectomía laparoscópica por puerto umbilical asistido*
}

\author{
Dr. NICOLÁS SOLANO V. ${ }^{1}$, Al. NICOLÁS SOLANO R. ${ }^{2}$ \\ 1 Clínica Los Leones de La Calera. \\ 2 Alumno de Medicina, Universidad de Valparaíso. \\ Valparaíso, Chile.
}

\begin{abstract}
Transumbilical laparoscopic cholecystectomy assisted with a second port. Experience in 60 patients
\end{abstract}

Background: Transumbilical laparoscopic surgery offers substantial esthetic benefits. The use of a second port to introduce a second trocar, reduces the need of special or articulated instruments. Aim: To report the experience with transumbilical cholecystectomy, assisted using a second port, to reduce technical complexity. Patients and Methods: The surgical technique was prospectively evaluated in 60 patients aged 18 to 77 years (39 women), that required a cholecystectomy. All had a surgical risk classified according to the American Society of Anesthesiologists (ASA) as I or II. Patients with gallbladder cancer, choledocholithiasis, were excluded. Two trocar (10 and $5 \mathrm{~mm}$ ) were introduced by transumbilical incision and other one $5 \mathrm{~mm}$ trocar was introduced in the right iliac fossa for $0^{\circ}$ frontal optics. Results: The mean operative time was 72 minutes (range 35-165). Fifty seven patients had a 24 h hospital stay. Two patients had to be converted to open surgery due to a complex pedicle dissection and one patient required an additional trocar. There were three complications. An umbilical seroma, a bile leakage through a Luschka duct, that was managed conservatively and one bile tract stenosis, that appeared four months after the operation and required surgical repair. Conclusions: Transumbilical cholecystectomy using a second additional port was a successful procedure, with similar esthetic results as single port surgery, but less costly.

Key words: Transumbilical surgery, cholecystectomy, laparoscopic surgery.

\section{Resumen}

Introducción: La cirugía laparoscópica evoluciona hacia técnicas menos invasivas, entre ellas la cirugía por puerto único umbilical, con mayor beneficio estético. El objetivo del presente trabajo es presentar los resultados de una técnica en colecistectomías laparoscópicas por puerto umbilical asistido por segundo puerto. La hipótesis planteada es que la técnica descrita es sencilla, segura y con beneficio estético similar al puerto único umbilical. Pacientes y Métodos: Técnica quirúrgica realizada prospectivamente en una serie de 60 pacientes ASA I y ASA II con consentimiento informado. Se incluye colecistolitiasis, pólipos, colecistitis aguda y crónica. Se excluye cáncer vesicular y coledocolitiasis. No se excluyeron casos por complejidad técnica o IMC. Se insertan 2 trocares de trabajo de 10 y $5 \mathrm{~mm}$ por incisión transumbilical y trocar de 5 mm en fosa

*Recibido el 24 de julio de 2011 y aceptado para publicación el 17 de octubre de 2011.

Correspondencia: Dr. Nicolás Solano V. Alberdi 797, Depto. N², Quillota, Chile. nicolassolanov@gmail.com 
iliaca derecha para óptica frontal de $0^{\circ}$. Resultados: Tiempo operatorio promedio de 72 minutos, mediana de 60 y extremos de 35 y 165. Tuvieron hospitalización de 24 horas 57 pacientes (95\%). Hubo 2 conversiones a cirugía abierta por difícil disección del pedículo y un caso requirió trocar adicional. Hubo 3 complicaciones: un seroma de ombligo, una bilirragia por conducto de Luschka manejado conservadoramente y 1 estenosis de vía biliar postoperatoria a 4 meses, con reparación quirúrgica. Conclusiones: La colecistectomía laparoscópica por puerto umbilical asistida, logra resultados exitosos y con similares ventajas estéticas que el puerto único. El bajo costo de nuestra técnica permite realizarla en hospitales públicos y centros privados con menores recursos.

Palabras clave: Colecistectomía laparoscópica, puerto único, SILS.

\section{Introducción}

Dentro de los propósitos de la cirugía laparoscópica se ha planteado el logro de mejores resultados estéticos en comparación con la cirugía convencional. En esa línea, se han desarrollado, gracias a los avances tecnológicos, técnicas cada vez menos invasivas, llegando a extirpar órganos a través de orificios naturales, sin cicatrices, por medio de cirugía endoscópica que utiliza como vía de trabajo y extracción de los órganos, la vagina, el estómago o el colon (NOTES) $)^{1-3}$. Como variante de esta técnica, se han desarrollado abordajes transumbilicales para la introducción de ópticas e instrumentos que permiten realizar diversas cirugías (NOTUS) ${ }^{2,4}$. Las técnicas de cirugía laparoscópica por puerto umbilical exclusivo aportan ventajas estéticas, pero no han mostrado diferencias en cuanto a dolor y a tiempo de estadía postoperatorio respecto de la cirugía laparoscópica convencional. Hasta ahora, usando esta técnica, se han realizado colecistectomías, apendicectomías, hemicolectomías derechas y gastrectomías verticales ${ }^{5-10}$. Estas técnicas generalmente usan dispositivos y trocares especiales para el acceso abdominal, acompañados de instrumentos laparoscópicos articulados ${ }^{2,11-17}$. Variantes de esta técnica son las cirugías con dos y tres puertos de entrada ${ }^{6,17-21}$.

El objetivo del presente trabajo es describir los resultados en una serie de casos personal con una técnica de colecistectomía laparoscópica realizada por puerto umbilical asistida con un trocar adicional de $5 \mathrm{~mm}$. El propósito es emular las ventajas estéticas del puerto único umbilical con una técnica quirúrgica sencilla y sin costos adicionales, ya que no utiliza dispositivos especiales ni instrumentos articulados. La ventaja económica de esta técnica permite realizarla en hospitales públicos o clínicas privadas con menores recursos.

La hipótesis de este trabajo es demostrar que la técnica diseñada es factible de realizar en forma segura, sin exclusiones por dificultades técnicas y que aporta los beneficios estéticos de la técnica de puerto umbilical único, ya que la incisión de $5 \mathrm{~mm}$ en fosa iliaca derecha es casi imperceptible, y sus tiempos quirúrgicos pueden acercarse a los de la técnica laparoscópica convencional, una vez superada la curva de aprendizaje.

\section{Pacientes y Método}

Se diseña un protocolo prospectivo para una serie de casos con la realización de una técnica de colecistectomía laparoscópica por puerto umbilical asistido por un segundo puerto de $5 \mathrm{~mm}$. El protocolo incluye a 60 pacientes con diversas patologías de la vesícula biliar diagnosticados por clínica y ecotomografía, los que son intervenidos entre septiembre de 2009 y junio de 2011. Las intervenciones fueron todas realizadas por el autor, en la clínica Los Leones de La Calera. Se obtuvo el consentimiento informado de todos los pacientes para la realización de esta técnica. Se obtuvo además la aprobación por parte del comité de ética de la clínica para realizar esta técnica quirúrgica y consultar las fichas de los pacientes con el propósito de revisar los resultados de la casuística de este trabajo.

Se analizan variables demográficas como sexo y edad de los pacientes. Se evalúa el tiempo operatorio total y el tiempo de estadía de hospitalización. Se describen los diagnósticos preoperatorios, y los diagnósticos postoperatorios. Se evalúa la existencia de conversión en esta casuística. Para tal efecto, se diferencian 2 tipos de conversión: a laparoscopia convencional con cuatro trocares o a cirugía abierta con laparotomía. Además, se analiza la necesidad de uso de trocar adicional (tercer puerto), sin convertir a laparoscopia convencional. No evaluamos dolor postoperatorio con esta técnica, por el hecho de no tener un grupo control de laparoscopia convencional que permita compararlo con la laparoscopia de puerto umbilical mediante un diseño aleatorio. Sin embargo, observamos que el dolor para esta técnica se presenta en un rango de 3 a 4 en la escala de EVA, semejante a lo que se describe para cirugía laparoscópica con cuatro puertos.

Criterios de inclusión: Pacientes portadores de 
patología de la vesícula biliar como colecistolitiasis, colecistitis aguda y crónica y pólipos vesiculares. Se incluyen pacientes sin descartarlos por su edad ni por su IMC. Los pacientes operados incluidos son ASA 1 y 2.

Criterios de exclusión: Todo paciente con sospecha o confirmación de diagnóstico de coledocolitiasis. El estudio se realiza por medio de la clínica (antecedente anamnéstico de ictericia y coluria, y/o hallazgos al examen físico), y con la información de la ecotomografía abdominal y pruebas hepáticas. Este criterio de exclusión es obviado en el caso de que se pueda contar con resolución endoscópica preoperatorio de la litiasis de la vía biliar. Debemos destacar que en nuestra región este recurso no es de fácil acceso.

También es criterio de exclusión la sospecha de cáncer de vesícula biliar.

Se elabora una base de datos en una planilla Excel y las variables a estudiar se analizan con Software Intercooled Stata 8.1.

La técnica quirúrgica consiste en la realización de una incisión de unos 15 mm transumbilical (Figura 1). Luego se realiza neumoperitoneo por medio de aguja de Veress. Alcanzada la presión intrabdominal de $15 \mathrm{~mm} \mathrm{Hg}$ se introduce por la incisión transumbilical un trocar de 10 y otro de $5 \mathrm{~mm}$. Ambos trocares, paralelos y transumbilicales están destinados a la introducción de los instrumentos quirúrgicos que son manejados por ambas manos del cirujano. Se utilizan trocares e instrumentos laparoscópicos tradicionales reutilizables. En fosa iliaca derecha, en posición baja, se introduce un trocar de $5 \mathrm{~mm}$ a través del cual se introduce una óptica de $5 \mathrm{~mm}$, de cero grado, frontal (Figura 2). La incisión para este segundo puerto es casi imperceptible debido a su tamaño y ubicación. El ayudante primero está encargado de llevar la cámara y considerando la técnica descrita, no se requeriría la presencia de un $2^{\circ}$ ayudante. La cirugía se inicia con la tracción del bacinete por el cirujano con una pinza; por el $2^{\circ}$ trocar se realiza la disección del pedículo con hook y pinza disector (Maryland) (Figura 3). El clipaje del conducto y arteria cística se realiza introduciendo clipadora por el trocar umbilical de $10 \mathrm{~mm}$, mientras el bacinete se tracciona con una pinza en trocar umbilical de $5 \mathrm{~mm}$. Se seccionan elementos del pedículo con tijera y se diseca lecho hepático con hook. Se realiza hemostasia y se extrae la vesícula por el acceso umbilical, el que se amplía comunicando los orificios de los trocares umbilicales. El uso de bolsa es selectivo para los casos de colecistitis aguda o ruptura intraoperatoria de vesícula. Se cierra el orificio umbilical ampliado con ácido Poliglactínico 0 y la piel con idéntico material 4/0 intradérmico. (Figura 4) No se realiza colangiografía intraoperatoria de rutina. Se utiliza profilaxis antibiótica con cefazolina 1 gr EV en dosis única administrada durante la inducción anestésica.

El seguimiento de los pacientes se hace por citaciones periódicas a consulta médica (1 semana y 1 mes). El resto del seguimiento se hace por vía telefónica.

Los primeros 50 pacientes tienen un seguimiento que va desde 5 a 22 meses. Los últimos 10 pacientes tienen un seguimiento desde 1 a 5 meses.

\section{Resultados}

La serie estuvo compuesta por 39 mujeres (65\%) y 21 varones (35\%). La edad promedio fue de 45,5 años, con una mediana de 46 y extremos de 18 y 77 años. Los diagnósticos preoperatorios abarcan toda la gama de patologías de la vesícula biliar tales como colecistolitiasis, colecistitis crónica y aguda, pólipos, vesícula alitiásica con pared engrosada, etc. El detalle de esta información se encuentra en la Tabla 1. Los diagnósticos postoperatorios son bastante similares a los preoperatorios y se detallan en la Tabla 2, siendo importante destacar que se encontraron 2 gangrenas vesiculares, 1 nódulo hepático en segmento cuatro, el cual se extirpó durante la misma laparoscopia y que resultó ser una hiperplasia nodular focal (HNF), y 2 cánceres de vesícula con compromiso seroso diagnosticados por la histopatología postoperatoria. Respecto a los tiempos quirúrgicos, el promedio fue de 72 minutos con una mediana de 60 minutos. El mínimo fue 35 minutos y el máximo 165 minutos. Sólo 2 pacientes de la serie (3,33\%) tuvieron un tiempo operatorio mayor de 130 minutos y 2 casos (3,33\%) superior a 150 minutos. Los mayores tiempos quirúrgicos corresponden a los primeros pacientes en la curva de aprendizaje inicial y a casos más complicados (gangrenas vesiculares, vesícula escleroatrófica). Los detalles se explican en la Tabla 3. Los días de estada hospitalaria se detallan en Tabla 4. Hubo 57 pacientes (95\%) que tuvieron 24 horas de estada hospitalaria; 1 paciente $(1,67 \%)$ tuvo 48 horas y 1 paciente $(1,67 \%) 72$ horas; ambos corresponden a los convertidos a cirugía abierta y que tenían cáncer vesicular con compromiso seroso (T3). Sólo 1 paciente $(1,67 \%)$ tuvo un tiempo mayor de hospitalización, de 7 días, correspondiendo a un paciente con gangrena vesicular que presentó bilirragia postoperatoria por conducto de Luschka el cual se manejó con drenaje en forma conservadora y tuvo buena evolución sin complicaciones posteriores.

Respecto a las conversiones, 57 pacientes (95\%) no requirieron de conversión a cirugía abierta ni a laparoscopia convencional de 4 trocares y la cirugía se logró realizar con éxito. En 2 casos (3,33\%), 


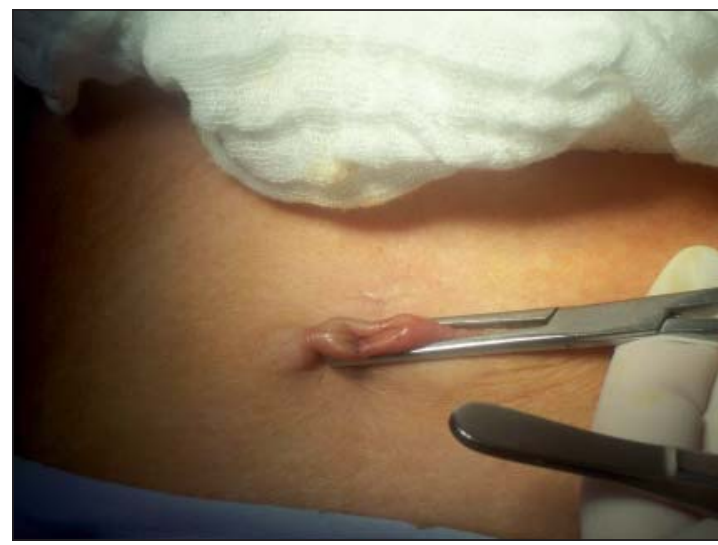

Figura 1. Se realiza incisión transumbilical.

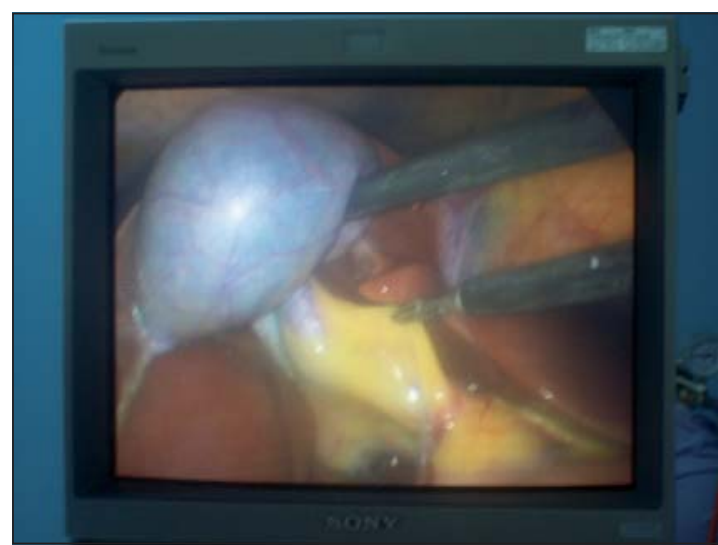

Figura 3. Disección de pedículo con una pinza, mientras la otra pinza sólo tracciona bacinete.

Tabla 1. Diagnósticos Pre-operatorios

\begin{tabular}{|lc|}
\hline Diagnóstico pre-operatorio & n (\%) \\
\hline Colecistolitiasis & $26(43,3)$ \\
\hline Colecistitis aguda litiásica & $13(21,67)$ \\
\hline Colecistitis crónica litiásica & $15(25)$ \\
\hline Colecistitis crónica litiásica escleroatrófica & $1(1,67)$ \\
\hline Pólipo vesicular & $2(3,33)$ \\
\hline Colecistitis crónica litiásica reagudizada & $2(3,33)$ \\
\hline $\begin{array}{l}\text { Colecistitis crónica alitiásica + } \\
\text { Engrosamiento de pared }\end{array}$ & $1(1,67)$ \\
\hline
\end{tabular}

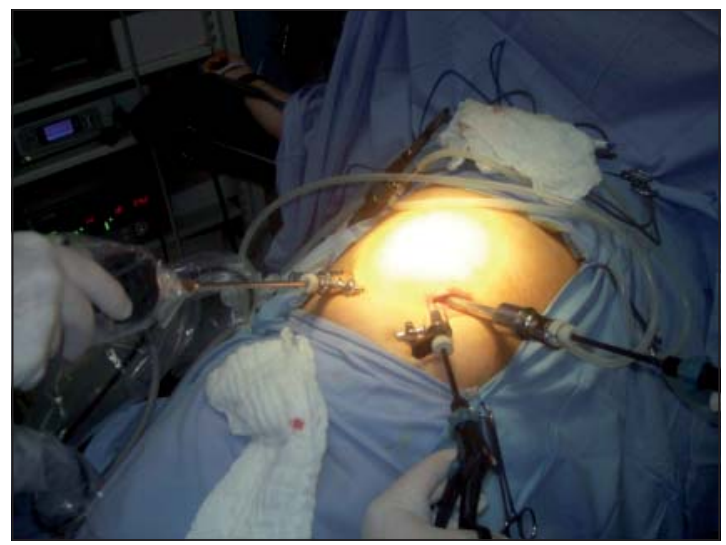

Figura 2. En puerto umbilical entran 2 trocares de 10 y 5 $\mathrm{mm}$. En FID entra cámara de $5 \mathrm{~mm}$ frontal $0^{\circ}$ por trocar de $5 \mathrm{~mm}$.

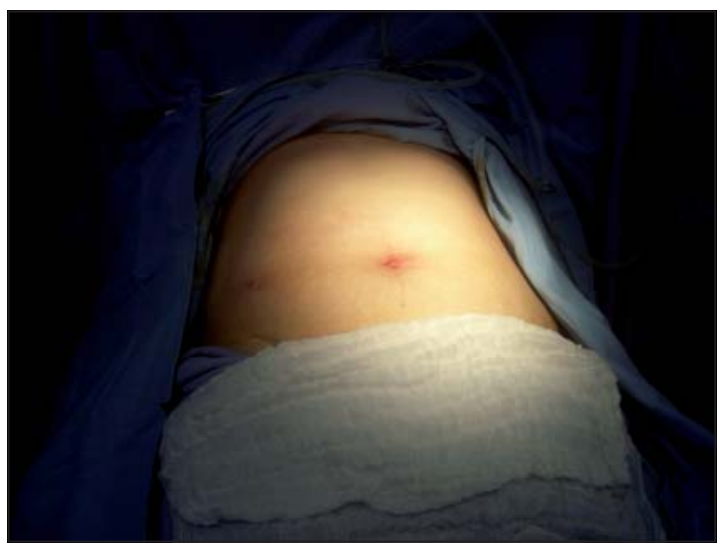

Figura 4. Aspecto final. Incisión transumbilical inaparente y la de FID muy pequeña y poco visible.

hubo conversión a cirugía abierta por gran dificultad técnica en la disección y corresponden a pacientes con cáncer de vesícula con compromiso seroso (T3) confirmados por la biopsia postoperatoria. En 1 caso (1,67\%), se requirió agregar un trocar adicional de apoyo por dificultades técnicas sin necesidad de conversión de ningún tipo. No hubo conversión a laparoscopia convencional de cuatro puertos.

Las complicaciones de la serie se indican en la Tabla 5. Un paciente $(1,67 \%)$ tuvo una onfalitis asociada a un seroma que se manejó en forma conservadora. Otro paciente $(1,67 \%)$ operado por una colecistitis aguda corresponde al paciente previamente descrito que desarrolló una bilirragia y que se manejó en forma conservadora con drenaje. Finalmente, una paciente de la serie presentó icte- 
Tabla 2. Diagnósticos y hallazgos post-operatorios

\begin{tabular}{|lcl|}
\hline Diagnóstico post-operatorio & n & (\%) \\
\hline Colecistolitiasis & $24(40)$ \\
\hline Colecistitis aguda litiásica & $9(15)$ \\
\hline Colecistitis crónica litiásica & $14(23,3)$ \\
\hline Colecistitis crónica litiásica escleroatrófica & 1 & $(1,67)$ \\
\hline Pólipo vesicular & 1 & $(1,67)$ \\
\hline Colecistitis crónica litiásica reagudizada & 3 & $(5)$ \\
\hline $\begin{array}{l}\text { Colecistitis aguda litiásica }+ \\
\text { Hernia umbilical }\end{array}$ & $1 \quad(1,67)$ \\
\hline Colecistitis aguda litiásica + Ca Vesícula T33 & $1 \quad(1,67)$ \\
\hline $\begin{array}{l}\text { Pólipo vesicular + Nódulo hepático } \\
\text { segmento } 4\end{array}$ & $1 \quad(1,67)$ \\
\hline Colecistolitiasis + Hidrops vesicular & $1 \quad(1,67)$ \\
\hline $\begin{array}{l}\text { Colecistitis aguda litiásica + } \\
\text { Ca Vesícula + Empiema vesicular }\end{array}$ & $1 \quad(1,67)$ \\
\hline $\begin{array}{l}\text { Colecistitis aguda litiásica + } \\
\text { Gangrena vesicular }\end{array}$ & $2(3,33)$ \\
\hline $\begin{array}{l}\text { Colecistitis crónica alitiásica + } \\
\text { Engrosamiento de pared }\end{array}$ & \\
\hline
\end{tabular}

ricia y dolor abdominal cuatro meses posterior a su colecistectomía; el estudio ecotomográfico y de colangioresonancia, demostró la existencia de una estenosis benigna de la vía biliar. La paciente fue intervenida encontrándose una estenosis del segmento medio del conducto hepático posiblemente de etiología térmica. No hubo secciones ni presencia de clips en la vía biliar. Se realizó una reparación mediante hepático yeyuno anastomosis en Y de Roux con muy buena evolución. No tenemos mortalidad operatoria.

\section{Discusión}

En relación a la realización de la colecistectomía por puerto único, encontramos en la literatura experiencias que seleccionan los pacientes por IMC (excluyen obesos) y por complejidad técnica (excluyen casos difíciles $)^{5,8,20,22-26}$. En nuestra serie hemos operado todo tipo de pacientes sin exclusión por IMC ni por complejidad de la patología biliar, por
Tabla 3. Tiempo operatorio

\begin{tabular}{|r|c|}
\hline Tiempo operatorio & $\mathbf{n}(\mathbf{\%})$ \\
\hline $30-49$ minutos & $12(20)$ \\
\hline $50-69$ minutos & $20(33,33)$ \\
\hline $90-109$ minutos & $10(16,67)$ \\
\hline $110-129$ minutos & $14(23,33)$ \\
$130-149$ minutos & $0(0)$ \\
$150-169$ minutos & $2(3,33)$ \\
\hline
\end{tabular}

Tabla 4. Tiempo de hospitalización

\begin{tabular}{|l|c|}
\hline Tiempo hospitalización & n (\%) \\
\hline 24 horas & $57(95)$ \\
48 horas & $1(1,67)$ \\
72 horas & $1(1,67)$ \\
168 horas (7 días) & $1(1,67)$ \\
\hline
\end{tabular}

Tabla 5. Morbilidad

\begin{tabular}{|lc|}
\hline Morbilidad & n (\%) \\
\hline Fístula biliar & $1(1,67)$ \\
\hline Onfalitis y seroma & $1(1,67)$ \\
\hline Estenosis tardía de Vía Biliar (4M) & $1(1,67)$ \\
\hline
\end{tabular}

lo que nuestros resultados nos parecen satisfactorios. Los tiempos quirúrgicos son más prolongados en los casos iniciales y en los cuadros más complejos tales como colecistitis aguda, gangrena vesicular y vesícula escleroatrófica. En la medida que avanzamos en la curva de aprendizaje, los tiempos quirúrgicos se reducen llegando hasta 35 minutos el mínimo con un promedio de 72 minutos y mediana de 60 minutos, lo que se puede considerar comparable al método laparoscópico tradicional. Rodríguez y $\operatorname{cols}^{5}$, publica una serie de 12 casos con tiempos de 160 y 45 minutos según curva de aprendizaje. La mayoría de nuestros pacientes (95\%) tuvo un período de hospitalización de 24 horas; los dos pacientes convertidos a cirugía abierta tuvieron 48 y 72 horas respectivamente y sólo 1 paciente (colecistitis aguda y gangrena vesicular con fístula biliar externa) tuvo un período de 7 días. Estos resultados están dentro de lo esperable. Rodríguez ${ }^{5}$, reporta hospitalización postoperatoria entre 12 y 36 horas. Algunos autores refieren hospitalizaciones de 24 ho- 
$\operatorname{ras}^{13,14,19,23,26-30}$, y otros comunican entre 1,5 y 2,5 días de estadía ${ }^{15,28,31-33}$. Encontramos autores con hospitalizaciones menores a un día 22,24,34,35. También se reporta mayor tiempo de hospitalización (3 a 5 días) asociado a casos complicados ${ }^{11,13,36}$. Nuestra técnica resulta exitosa en un 95\% con un porcentaje de conversión a cirugía abierta de 3,33\% (2 pacientes) debido a la existencia de una disección muy difícil y que resultaron ser cánceres de vesícula biliar con compromiso seroso. No hubo conversión a laparoscopia con 4 puertos. Sólo 1 paciente (uno de los 2 con gangrena vesicular y bilirragia postoperatoria) requirió asistencia de un tercer puerto por dificultades técnicas y no fue necesario convertir. Existen series con selección de pacientes cuyos casos no son complicados y ellos no reportan conversiones ${ }^{5,23,26}$. En nuestra experiencia no hubo exclusión de pacientes con patología complicada y el porcentaje de conversión puede considerarse bajo.

Encontramos en nuestra serie un nódulo hepático en el segmento cuatro cuya biopsia informó una hiperplasia nodular focal. El nódulo se extirpó exitosamente en forma laparoscópica usando la misma vía de puerto umbilical asistido, sin necesidad de agregar otros trocares o de convertir la técnica.

Respecto de las complicaciones quirúrgicas, en nuestra experiencia reportamos 3 casos; dos precoces y uno más tardío. Dentro de las precoces, tenemos una onfalitis y seroma que se resuelve sin problemas. La literatura reporta con frecuencia este evento $^{12,23,28,32,38,39}$. Otra complicación precoz que tuvimos es una bilirragia postoperatoria por conducto de Luschka, manejado conservadoramente sólo con drenaje y que se resuelve al quinto día postoperatorio. En la literatura se mencionan fístulas biliares por filtración del conducto cístico o por conductos de Luschka que se resuelven con drenaje o por intervención endoscópica ${ }^{10,12,17,24,29,32,35,37}$. Nuestra tercera complicación fue más tardía, detectada cuatro meses después de la cirugía y consistió en la aparición de una estenosis benigna de la vía biliar, posiblemente de causa térmica originando cicatrización retráctil. Esta complicación se intentó resolver en forma endoscópica, sin éxito, por lo que se operó realizando una derivación biliodigestiva con buen resultado hasta la fecha. En la reoperación no se evidenció clips cerca de la vía biliar ni sección de ella. En otras experiencias, los autores reportan esta complicación. Kuon Lee et al ${ }^{15}$, informa una lesión de conducto hepático derecho en una experiencia de 37 casos, y Curcillo et al $^{32}$, comunica una estenosis de la vía biliar un año después de la cirugía en una experiencia de 297 casos.

Se describe en la literatura otras complicaciones que en nuestra serie no hubo tales como hemoperitoneo, lesión mesentérica y lesión intestinal ${ }^{5,15,25}$.
La literatura no reporta mortalidad operatoria con la técnica de colecistectomía por puerto único umbilical. En nuestra serie no hubo mortalidad relacionada con la técnica quirúrgica.

\section{Conclusión}

La técnica de colecistectomía laparoscópica por puerto umbilical asistido por puerto adicional de 5 $\mathrm{mm}$ en fosa ilíaca derecha es una modalidad de la cirugía por puerto umbilical exclusivo, la cual conserva las ventajas estéticas de esta última debido a que el puerto adicional por su tamaño e inserción abdominal baja es prácticamente imperceptible. Usamos trocares e instrumental laparoscópico convencional reutilizable con lo cual no hay costos adicionales, lo que es una ventaja, pudiendo esta técnica realizarse en hospitales públicos y clínicas privadas con menores recursos. Nuestros resultados confirman la hipótesis de trabajo, ya que la colecistectomía laparoscópica por puerto umbilical asistido permite realizar una cirugía en forma exitosa y segura, con una morbilidad baja y dentro de lo esperable para cirugía de la vesícula biliar, tomando en consideración que se operaron pacientes no excluidos por IMC ni por dificultades técnicas. Los tiempos quirúrgicos son razonables y una vez superada la curva de aprendizaje terminan siendo semejantes a los de la laparoscopia convencional con cuatro puertos.

\section{Agradecimientos}

Agradezco al Dr. Jorge León Celsi por su permanente apoyo en el desarrollo de mi actividad quirúrgica y al Dr. Xavier De Aretxabala Urquiza igualmente por su apoyo en mi actividad quirúrgica y el análisis crítico de este artículo de investigación quirúrgico.

\section{Referencias}

1. Lucena J, Coronel P, Useche C. Cirugía endoscópica transorificios naturales (NOTES). Rev Chil Cir. 2009;61:317-28.

2. Nguyen NT, Reavis KM, Hinojosa MW, Smith BR, Wilson SE. Laparoscopic transumbilical cholecystectomy without visible abdominal scars. J Gastrointest Surg. 2009;13:1125-8.

3. Willingham FF, Brugge WR.Taking NOTES: translumenal flexible endoscopy and endoscopic surgery. Curr Opin Gastroenterol. 2007;23:550-5.

4. Jacob DA, Raakow R. Single-port transumbilical endoscopic cholecystectomy: a new standard? Dtsch Med Wochenschr. 2010;135:1363-7. 
5. Rodríguez J, Vial G, Herrera R, Araneda T. Colecistectomía laparoscópica transumbilical. Una técnica reproducible. Rev Chil Cir. 2010;62:33-6.

6. Rocha M. Colecistectomía por laparoscopia: abordaje con dos incisiones de entrada. Rev Chil Cir. 2010;62:836.

7. Arias F, Prada NE. Apendicectomía y colecistectomía "invisibles": cirugía totalmente laparoscópica por un puerto umbilical (OPUS). Rev Chil Cir. 2009;61:181-6.

8. López F, Capona R, Heine C, Wainstein C, Márquez N. Hemicolectomía derecha laparoscópica por puerto único para cáncer de colon. Rev Chil Cir. 2009;61:566-70.

9. Maluenda F, León J, Turu I, Cavalla C, Abarca J, Monres J, y cols. Gastrectomía vertical laparoscópica transumbilical. Rev Chil Cir. 2010;62:576-81.

10. Chow A, Purkayastha S, Paraskeva P. Appendicectomy and cholecystectomy using single-incision laparoscopic surgery (SILS): the first UK experience. Surg Innov. 2009;16:211-7.

11. Misiak A, Szczepanik AB. Laparoscopic cholecystectomy with single incision laparoscopic surgery. Pol Merkur Lekarski 2009;27:372-4.

12. Garijo Alvarez J, Sánchez López JD, González Elosua T, Gascon Hove M, García-Sancho Téllez L, Del Castillo Diez F, et al. Laparoscopic transumbilical cholecystectomy. Results with the gel device and literature review. Cir Esp. 2010;87:293-8.

13. Fumagalli U, Verrusio C, Elmore U, Massaron S, Rosati R. Preliminary results of transumbilical single-port laparoscopic cholecystectomy. Updates Surg. 2010;62:105-9.

14. MacDonald ER, Alkari B, Ahmed I. Single-port laparoscopic cholecystectomy-the Aberdeen technique. Surg Laparosc Endosc Percutan Tech. 2010;20:7-9.

15. Kuon Lee S, You YK, Park JH, Kim HJ, Lee KK, Kim DG. Single-port transumbilical laparoscopic cholecystectomy: a preliminary study in 37 patients with gallbladder disease. J Laparoendosc Adv Surg Tech. 2009;19:495-9.

16. Ito M, Asano Y, Horiguchi A, Shimizu T, Yamamoto T, Uyama I, et al. Cholecystectomy using single-incision laparoscopic surgery with a new SILS port. J Hepatobiliary Pancreat Sci. 2010;17:688-91.

17. Kravetz AJ, Iddings D, Basson MD, Kia MA. The learning curve with single-port cholecystectomy. JSLS 2009;13:332-6.

18. Vega GR, Preciado CY, Becerril RA, Serrano V, Solórzano DA, Su Gandarilla J. Colecistectomía laparoscópica con tres puertos. Una modificación al abordaje. Rev Mex Cir Endoscop. 2003;4:134-40.

19. Dávila F, Dávila U, Montero JJ, Lemus J, López FX, Villegas J. Colecistectomía laparoscópica con un solo puerto visible subxifoideo de $5 \mathrm{~mm}$. Rev Mex Cir Endoscop. 2001;2:16-20.

20. Kagaya T. Laparoscopic cholecystectomy via two ports, using the Twin-Port system. J Hepatobiliary Pancreat Surg. 2001;8:76-80.
21. Podolsky ER, Curcillo PG. Reduced-port surgery: preservation of the critical view in single-port-access cholecystectomy. Surg Endosc. 2010;24:3038-43.

22. Martín Fernández J, Jara Sánchez A, Manzanares Campillo MC, Menéndez Sánchez P, Muñoz Atienza V, Padilla Valverde D, et al. Single port laparoscopic cholecystectomy in major ambulatory surgery (MAS). Cir Esp. 2010;88:328-31.

23. Rawlings A, Hodgett SE, Matthews BD, Strasberg SM, Quasebarth M, Brunt LM. Single-incision laparoscopic cholecystectomy: initial experience with critical view of safety dissection and routine intraoperative cholangiography. J Am Coll Surg. 2010;211:1-7.

24. Solomon D, Bell RL, Duffy AJ, Roberts KE. Single-port cholecystectomy: small scar, short learning curve. Surg Endosc. 2010;24:2954-7.

25. Romanelli JR, Roshek TB, Lynn DC, Earle DB. Singleport laparoscopic cholecystectomy: initial experience. Surg Endosc. 2010;24:1374-9.

26. Rao PP, Bhagwat SM, Rane A. The feasibility of single port laparoscopic cholecystectomy: a pilot study of 20 cases. HPB (Oxford). 2008;10:336-40.

27. Yu WB, Zhang GY, Li F, Yang QY, Hu SY. Transumbilical single port laparoscopic cholecystectomy with a simple technique: initial experience of 33 cases. Minim Invasive Ther Allied Technol. 2010;19:340-4.

28. Podolsky ER, Rottman SJ, Curcillo PG. Single port access (SPA) cholecystectomy: two year follow-up. JSLS 2009;13:528-35.

29. Edwards C, Bradshaw A, Ahearne P, Dematos P, Humble $\mathrm{T}$, Johnson R, et al. Single-incision laparoscopic cholecystectomy is feasible: initial experience with 80 cases. Surg Endosc. 2010;24:2241-7.

30. Erbella J Jr, Bunch GM. Single-incision laparoscopic cholecystectomy: the first 100 outpatients. Surg Endosc. 2010;24:1958-61.

31. Al-Tayar H, Nielsen PE, Jorgensen LN. Transumbilical cholecystectomy. Ugeskr Laeger. 2010;172:1508-11.

32. Curcillo PG, Wu AS, Podolsky ER, Graybeal C, Katkhouda N, Saenz A, et al. Single-port-access (SPA) cholecystectomy: a multi-institutional report of the first 297 cases. Surg Endosc. 2010;24:1854-60.

33. Hong TH, You YK, Lee KH. Transumbilical single-port laparoscopic cholecystectomy: scarless cholecystectomy. Surg Endosc. 2009;23:1393-7.

34. Bucher P, Pugin F, Buchs N, Ostermann S, Charara F, Morel P. Single port access laparoscopic cholecystectomy (with video). World J Surg. 2009;33:1015-9.

35. Roberts KE, Solomon D, Duffy AJ, Bell RL. Singleincision laparoscopic cholecystectomy: a surgeon's initial experience with 56 consecutive cases and a review of the literature. J Gastrointest Surg. 2010;14:506-10.

36. Hirano Y, Watanabe T, Uchida T, Yoshida S, Tawaraya $\mathrm{K}$, Kato $\mathrm{H}$, et al. Single-incision laparoscopic cholecystectomy: single institution experience and literature review. World J Gastroenterol. 2010;16:270-4. 
37. Allemann P, Schafer M, Demartines N. Critical appraisal of single port access cholecystectomy. Br J Surg. 2010;97:1476-80.

38. Wen KC, Lin KY, Chen Y, Lin YF, Wen KS, Uen YH. Feasibility of single-port laparoscopic cholecystectomy using a homemade laparoscopic port: a clinical report of 50 cases. Surg Endosc. 2011;25:879-82.

39. Elsey JK, Feliciano DV. Initial experience with singleincision laparoscopic cholecystectomy. J Am Coll Surg. 2010;210:620-6.

40. Chang SK, Tay CW, Bicol RA, Lee YY, Madhavan $\mathrm{K}$. A case-control study of single-incision versus standard laparoscopic cholecystectomy. World J Surg. 2011;35:289-93.
41. Carr A, Bhavaraju A, Goza J, Wilson R. Initial experience with single-incision laparoscopic cholecystectomy. Am Surg. 2010;76:703-7.

42. Fronza JS, Linn JG, Nagle AP, Soper NJ. A single institution's experience with single incision cholecystectomy compared to standard laparoscopic cholecystectomy. Surgery 2010;148:731-6.

43. Duron VP, Nicastri GR, Gill PS. Novel technique for a single-incision laparoscopic surgery (SILS) approach to cholecystectomy: single-institution case series. Surg Endosc. 2011;25:1666-71.

44. Langwieler TE, Nimmesgern T, Back M. Single-port access in laparoscopic cholecystectomy. Surg Endosc. 2009;23:1138-41. 